\title{
Differential expression of the IRF6 gene in the signs of Van der Woude Syndrome: Are distinct genetic modifiers operating?
}

\author{
Rosany de Oliveira Lisboa ${ }^{1,7,9}$, Cláudia Maria da Rocha Martins ${ }^{5}$, Maria Elisabete Silva Santos ${ }^{1,8}$, Danilo Leôncio Aguiar \\ Pereira $^{1,8}$, Flávia Martinez de Carvalho ${ }^{4}$, leda Maria Oriolii, ${ }^{2,3}$, João Farias Guerreiro ${ }^{6,8}$, Alexandre Rezende Vieira ${ }^{7}$, Luiz Carlos \\ Santana-da-Silva ${ }^{1,9,10}$ \\ ${ }^{1}$ Laboratory of Inborn Errors of Metabolism, Institute of Biological Sciences, Federal University of Pará, Pará, Brazil. \\ ${ }^{2}$ Department of Genetics, Institute of Biology, Federal University of Rio de Janeiro, Rio de Janeiro, Brazil. \\ ${ }^{3}$ ECLAMC (Latin-American Collaborative Study of Congenital Malformations) at INAGEMP (National Institute of Population Medical Genetics), Rio \\ de Janeiro, Brazil. \\ ${ }^{4}$ ECLAMC at LEMC (Laboratory of Congenital Malformation Epidemiology), Oswaldo Cruz Institute, FIOCRUZ, Rio de Janeiro, Brazil. \\ ${ }^{5}$ Department of Speech therapy, Ophir Loyola Hospital, Pará, Brazil. \\ ${ }^{6}$ Laboratory of Human and Medical Genetics, Federal University of Pará, Pará, Brazil. \\ ${ }^{7}$ Departments of Oral Biology and Pediatric Dentistry and Center for Craniofacial and Dental Genetics, School of Dental Medicine, University of \\ Pittsburgh, Pittsburgh, Pennsylvania, USA. \\ ${ }^{8}$ Genetics and Molecular Biology Postgraduate Studies Program, Federal University of Pará, Pará, Brazil. \\ ${ }^{9}$ Oncology and Medical Sciences Graduate Program, Federal University of Pará, Pará, Brazil. \\ ${ }^{10}$ National Institute on Population Medical Genetics, Institute of Biological Sciences, Federal University of Pará, Pará, Brazil.
}

\section{Abstract}

Objective: The purpose of this study was to report a new variant in the Interferon Regulatory Factor 6 gene (IRF6) and to determine phenotype-genotype correlations in a family segregating Van der Woude syndrome.

Methods: A five-generation family of 80 individuals segregating VWS was investigated using a tabulated pedigree but considering that three individuals registered in the pedigree died shortly after birth, the final sample size was 77 individuals. Five individuals had a complete dental clinical examination and molecular analysis performed using direct sequencing of the exon 4 and an adjacent region with 23 base pairs of the IRF6 gene.

Results: Features of VWS reported in family history were present in $36.4 \%$ (28/77) of all family members; of these $57 \%(16 / 28)$ had pits in the lower lip, $36 \%(10 / 28)$ had both pits and orofacial clefts and $7 \%$ $(2 / 28)$ had only orofacial clefts. Developmental dental anomalies were observed in three individuals. The sequence analysis of exon 4 of the IRF6 gene carried out for 4 family members revealed the presence of the SNP rs7552506 (c.175-5C> G) located in five base pairs before the start of exon. The analysis of exon 4 of the IRF6 gene also revealed a new variant c.269G $>C$ (p.Ser90Thr) which causes exchange of the Serine amino acid residue for the Threonine residue.

Conclusion: The c.269G $>C$ (p.Ser90Thr) can interfere with multimeric interactions and with protein conformation that will be slightly destabilized, because the mutant residue is bigger than the wild-type residue. The phenotypic variations in the cases studied, despite carrying the same genetic mutation, suggest that distinct genetic modifiers operate on the formation of clefts and dental development.

Keywords: Van der Woude syndrome; Orofacial clefts; IRF6 gene

Citation: Lisboa RO, et al. (2021) Differential expression of the IRF6 gene in the signs of Van der Woude Syndrome: Are distinct genetic modifiers operating? Dentistry 3000. 1:a001 doi:10.5195/d3000.2021.164 Received: March 30, 2021 Accepted: August 5, 2021 Published: November 4, 2021

Copyright: @2021 Lisboa RO, et al. This is an open access article licensed under a Creative Commons Attribution Work 4.0 United States License.

Email: Icsantana-pa@hotmail.com 
Vol 9 No 1 (2021) DOI 10.5195/d3000.2021.164

\section{Introduction}

Orofacial clefts may occur in association with other birth defects or be isolated. The Van der Woude Syndrome (VWS) (Online Mendelian Inheritance in Man [OMIM]119300) is the most common and well-known syndrome associated with orofacial clefts, being present in approximately $2 \%$ of orofacial cleft cases [1, 2]. VWS consists of a rare congenital malformation and presents clinical features in addition to orofacial clefts, such as lower lip pits and occasionally, hypodontia. The frequency of VWS in the population varies from 1 : 100,000 to $1: 40,000$, and there are no reports of differences between males and females $[3,4]$.

The expression of VWS is highly variable, both interfamilial and within the same family. In other words, there is a range of associated phenotypes, from the complete absence of visible abnormalities, only the presence of pits in the lower lip, to the combination of these pits with different types of clefts. These types may include aspects such as laterality (unilateral or bilateral), extension (complete and incomplete) and may involve lip, palate or both $[2,5-7]$.
The etiology of VWS has been attributed to mutations in the IRF6 gene (Interferon Regulatory Factor 6). However, from a molecular point of view, mutations in the IRF6 gene can explain $70 \%$ of the cases of VWS. Several studies also point to the possibility of other genes causing VWS, despite the limitations of molecular biology techniques in identifying mutations in many of these genes [8-10].

The IRF6 gene is located on the long arm of chromosome 1 (1q32.3 - q41) and contains 10 exons; exons 1, 2 and 10 are not translated. The product encoded by the IRF6 gene is a 467 amino acid polypeptide $[8,11,12]$.

In contrast to the other members of the IRF family (interferon regulating factors), IRF6 does not participate in the innate immune response, however it is essential for embryonic development, can act as a tumor suppressor and participate in the differentiation of ectodermal tissues [13-15]. Recently, a new role assigned to the IRF6 ortholog gene is participation in the development of the exocrine gland. This information can be used to investigate the suspicion of disorders in the salivary glands of patients with the two forms of syndrome caused by mutations in the IRF6 gene, the VWS and Popliteal Pterygium Syndrome (SPP), as well as to understand the presence of dental problems, for example, caries, in patients with orofacial clefts [16].

The aim of this study was to report a new variant in the IRF6 gene and to determine phenotype-genotype correlations in a family segregating Van der Woude syndrome.

\section{Material and Methods}

\section{Genealogical data}

The family participating in the study was located in the Service of Assistance to the Patients with Orofacial Clefts at Ophir Loyola Hospital (Belém - Pará, Brazil). At the time, five members of this family were present, a mother and four children (two girls and two boys). All five had congenital pits in the lower lip and only one of them did not present orofacial cleft but instead presented excess of lower lip. The research was carried out in accordance with the basic ethical principles of the guidelines and standards that regulate research in human beings: autonomy, beneficence, non-maleficence and justice. The project was previously approved by the Ethics Committee of the Health Science Center of the 
Federal University of Pará (CAAE 4879.0.000.073-10, no140/10).

To obtain genealogical data, the tabulated pedigree method according to Polleta et al. (2014) [17] was used. The graphical construction of the pedigree was performed using an online tool from the Progeny program, available at:

www.progenygenetics.com.

Information about other members of this family was obtained during three interviews at different times, resulting in the registration of 80 individuals in the pedigree, belonging to five generations of the index family. It was not possible to specify the gender and the presence of any manifestations of VWS in only three cases, all of whom had died shortly after birth; of these three cases, two were Siamese twin siblings. Of the final sample size of 77 individuals analyzed, 36 (45\%) were females and 41 (51\%) were males (Figure 1).

\section{Phenotypic characteristics}

Family members with one or more phenotypic characteristics of VWS were considered affected individuals. These characteristics included cleft lip with or without palate, cleft palate, and the presence of pits, whether bilateral or unilateral. The types of orofacial clefts found were grouped into: cleft lip (CL), cleft lip with cleft palate (CLP) and cleft palate (CP). Dental information was gathered through a complete dental clinical examination.

\section{DNA analysis}

Four family members underwent molecular analysis of exon 4 of the IRF6 gene. The samples of biological material, 3 to $4 \mathrm{~mL}$ of blood, were collected through venipuncture and transferred to test tubes containing EDTA. For the extraction of DNA, the Invirsob spin blood mini kit (Invitek) was used. For the amplification of the sequence of interest in the polymerase chain (Polymerase Chain Reaction, PCR), the primers used were forward GCTCTGGGCAATGATAGGAC and reverse - ACCTTCTCCCCAGCACCT. The PCR products were subjected to direct sequencing analysis of exon 4 of IRF6 and its exon-intron boundaries based on capillary electrophoresis, using the $A B I$ PRISM BigDye kit, Terminator Cycle Sequencing. The same primers were used for PCR reactions and the $A B I$ PRISM $^{\circledR}$ 3500 Genetic Analyzer Capillary Sequencer by Applied Biosystems.

\section{Data analysis}

The family history information obtained from the tabulated pedigree was inserted in the spreadsheet of the Microsoft Excel 2010 program and later analyzed with the aid of the PEDINFO program available at S.A.G.E. (Statistical analysis for genetic epidemiology) version 6.3 (2012) (http://darwin.cwru.edu). The analysis of the occurrence of polymorphic mutations or variations in exon 4 of the IRF6 gene was performed using the BioEdit program version 7.1.9 (http://www.mbio.ncsu.edu/BioEd it/bioedit.html). For the in silico prediction of functional effects on the protein, the bioinformatics tools HOPE (http://www.cmbi.ru.nl/hope) [18], Provean (available at: http: // provean.jcvi.org/index.php) [19], SIFT (available at: http://sift.jcvi.org/) [20] and PolyPhen-2 (available at: http://genetics.bwh.harvard.edu/ pph2/) [21] were utilized. The data obtained was analyzed using descriptive statistics.

\section{Results}

The family did not present a history of consanguineous marriage and from the second generation on, where a family member has a cleft lip and pits, all subsequent generations have more than one affected relative. It is observed through the pedigree that the affected individuals belong only to the paternal, ascending and descending sides in relation to the index case 
Vol 9 No 1 (2021) DOI 10.5195/d3000.2021.164

(indicated with an arrow in the pedigree, Figure 1).
Lip pits were present in 26 of the 28 affected individuals and 12 out of 28 individuals had orofacial clefts, with six of them having the lip and palate affected. Ten individuals had both pits and orofacial clefts (Table 1).
Out of the five individuals that had a dental examination (Table 2), one had permanent dentition and four had primary dentition. Three out of five had developmental dental anomalies.

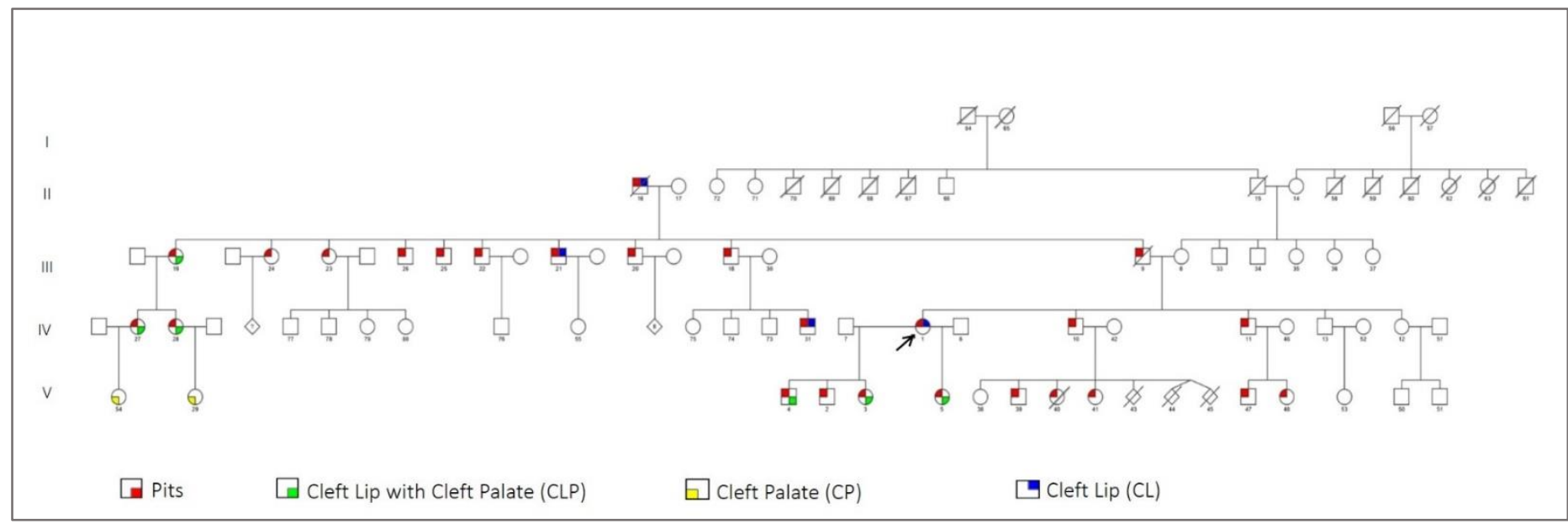

Figure 1. Pedigree showing family members affected by Van der Woude Syndrome.

Table 1. Phenotypic characteristics of Van der Woude Syndrome present in affected family members.

\begin{tabular}{lll}
\hline Phenotype & $\mathrm{n}$ & $\%$ \\
\hline Pits & 16 & 57 \\
Pits and clef lip & 4 & 14 \\
$\begin{array}{l}\text { Pits and cleft lip with cleft } \\
\text { palate }\end{array}$ & 6 & 21 \\
& & \\
Cleft palate & 2 & 7 \\
\hline Total & 28 & 100 \\
\hline
\end{tabular}


Table 2. Phenotype variation of Individuals with IRF6 Ser90Thr variant and Van der Woude syndrome.



*Biological sample not collected. 
The sequencing analysis of exon 4 of the IRF6 gene, performed for 4 family members (Table 2 ), revealed the presence of the SNP rs7552506 (NM_006147.3: c.175$5 C>G$ ), located five base pairs before exon and revealed a new variant c.269G>C (p.Ser90Thr) present in heterozygosity in the 4 affected family members (Figure 2).

The in silico analysis using the PROVEAN tool, pointed out that the c.269G > C variant (p.Ser90Thr) should be considered as deleterious since this variant had a score of -2.704; according to the platform parameters, a variant below the score of -2.5 is considered deleterious. As for the prediction result obtained by the Poliphen-2 tool, the new variant was considered potentially deleterious, with a score of 1,000 (sensitivity: 0.00; specificity: 1.00). According to the SIFT tool this variant is predicted as tolerated with a score of 0.37 .
One of the most informative prediction tools was HOPE. The analysis derived from it provided information about the structure of the mutant amino acid residue compared to the wild type of residue, showing that the mutant residue is structurally larger, a factor that can interfere in the multimeric interactions and in the conformation of the protein (Figure 3).

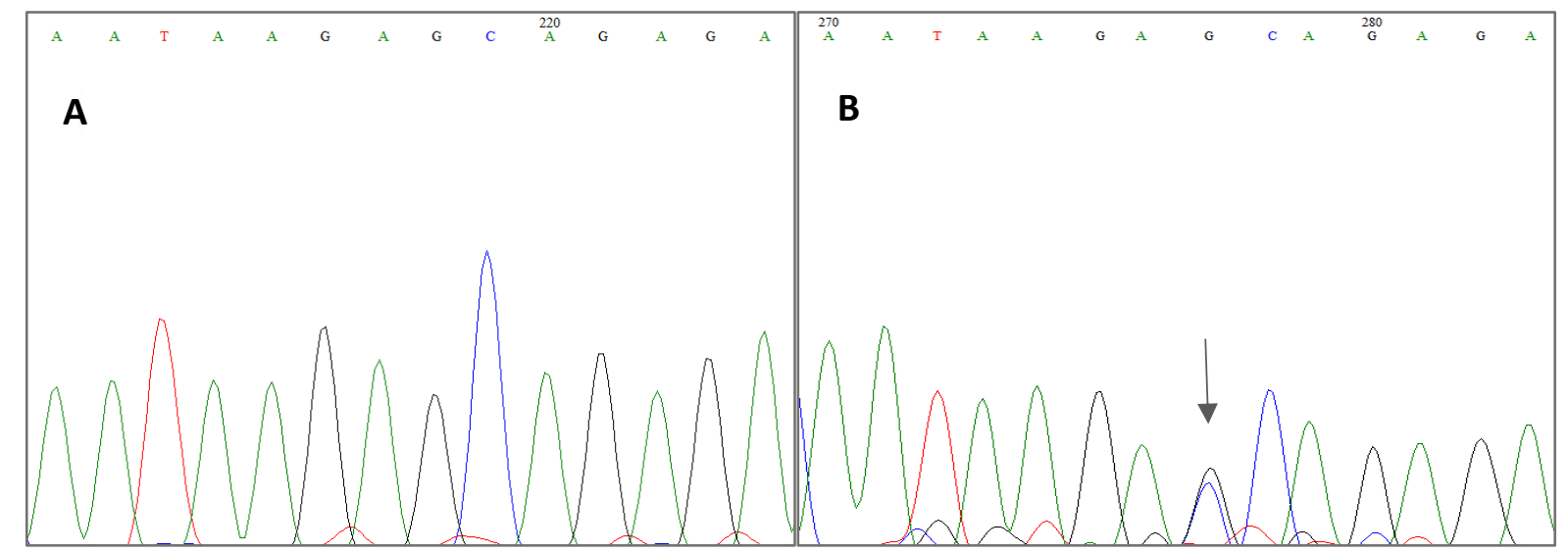

Figure 2. Electropherograms showing sequence without variant $(A)$ and with the new mutation in exon 4 of the IRF6 gene (B). An arrow indicates the altered nucleotide.

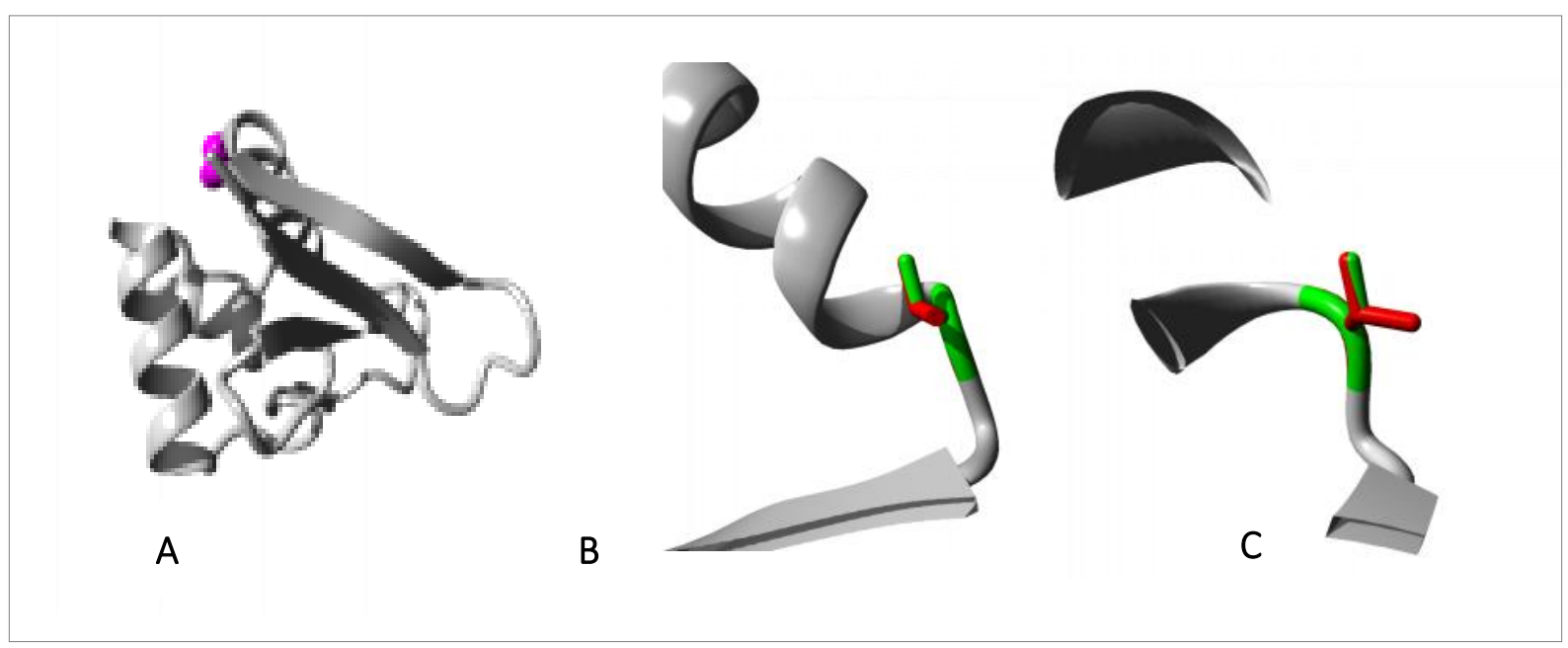

Figure 3. Images representing parts of the protein structure with the mutant residue. A. Ribbon overview of the protein, in gray, with the mutant residue represented in purple. B and C. Detailed view of the representation of the side chains of the mutant amino acid residue, in red compared to the wild residue, in green. Source: generated by the HOPE tool report. 


\section{Discussion}

For the clinical diagnosis of VWS, lower lip pits must be present in one or more members of a family, together with the presence of cleft lip with or without cleft palate and cleft palate [22]. The family investigated in this study presented both the classic VWS triad and mixed types of clefts. Compared to other families, the percentage of individuals affected in the studied family (36.4\%, 28/77) differs little from the percentages found by MartelliJúnior et al. (2007) [23] who studied two Brazilian families with VWS, one with 54 and the other with 17 descendants, where the phenotypic characteristics of the syndrome were present in $22.23 \%$ and $47.06 \%$ of the family members, respectively.

Due to the fact that in each generation of the studied family there is at least one affected member and they also had at least one of their parents affected, it's clear that this family follows the expected high penetrance that can vary from $80 \%$ to $100 \%$ according to previous studies in the literature $[2,24]$.

Variable expression was observed in the studied family, with the main phenotypic manifestation of VWS in the affected individuals being congenital lip pits. According to the literature, this type of pits occurs in around $85 \%$ of individuals affected by VWS and in $64 \%$ of cases it is the only manifestation of the syndrome [4, 7]. Regarding orofacial clefts, the studied family presented mixed types of clefts, with the most prevalent type (50\%) being the CLP, a finding similar to other studies $[2,7,25]$.

The presence of mixed types of orofacial clefts in the same family is often observed in families with VWS. In an attempt to explain this occurrence, it has been proposed that individuals with genetic components that cause VWS are subject to some type of multifactorial influence, similar to the non-syndromic form of orofacial clefts [8, 26-28]. Furthermore, another possible explanation could be the fact that another gene could be involved in this difference, for example variants in the gene GRHL3 (Grainyhead Like Transcription Factor 3) are more likely to have $\mathrm{CP}$ and less likely to have $\mathrm{CL}$ or lip pits than individuals with an IRF6 mutations [10]

As for the molecular analysis in exon 4, the SNP rs7552506 identified in the investigated family members is a polymorphism already described in the literature in studies with families with non-syndromic orofacial clefts and also in studies with families with VWS $[28,29]$. In the study by Pegelow et al. (2008) [30] with 17 Swedish families this SNP showed an association with CLP. In addition, in the same study it was found that when there was combined unilateral and bilateral CLP in the same family, there was an association of this polymorphism. This information corroborates the findings in the present study.

According to the analysis performed by the HOPE tool, the c.269G > C variant (p.Ser90Thr) found in the present study results in a mutant amino acid residue that is larger than the wild type residue, which can influence the conformation of the protein that will be slightly destabilized and, in addition, there may be disturbances in the multimeric interactions. The Ser90 position of the polypeptide chain is located in the DNA-binding domain (structural motif of the helix-gyrohelix type), which is important for the binding of other molecules and is in contact with the residue of a regulatory domain. Therefore, this mutation can affect this interaction and disrupt protein regulation. Variants that occur in the IRF6 gene are mostly missense-type and occur mainly in 
the DNA-binding or proteinbinding domains; variants in these domains are critical to the function of the IRF6 gene [8, 29, 31]. In addition, the silico analysis to verify the effect on the protein conformation of IRF6, suggests that this mutation is potentially pathogenic.

More specifically, the wild-type residue interacts with the amino acid residue Alanine at position 86 of the polypeptide chain; the size difference observed in the mutant residue prevents it from being in the correct position to perform the hydrogen bonding possible in the wild type of residue. Only two mutations for position 90 of the protein chain encoded by the IRF6 gene are described in the literature, one of which is mutation c. A268G (p.Ser90Gly) as described by Kondo et al. (2002) [8] that causes an exchange of the amino acid residue Serine for the amino acid residue Glycine. The other mutation, c.G269T ( $p$. Ser90I12) as described by Lima et al. (2009) [29], promotes the exchange of the amino acid residue Serine for the amino acid residue Isoleucine.

Among the dental anomalies associated with VWS, the most common among patients is hypodontia [32]. In our study, individuals who presented dental anomalies, as tooth agenesis were in the mandibular arch, which is something already predicted in the literature. Syndromic agenesis generally appears associated with other manifestations such as microdontia and the upper lateral incisor is the most frequently affected tooth in the cleft area $[33,34]$. Considering the data shown in Table 2, it's possible to see the phenotypic variation of the cases presented despite carrying the same genetic mutation, suggesting that distinct genetic modifiers operate on the formation of clefts and the dental development.

Despite being one of the most studied syndromic forms of orofacial clefts, this study reveals how much remains to be discovered in terms of how genetic factors act underlying the phenotypic expression of VWS. In addition, we emphasize that families with this syndrome still go unnoticed in health systems. Observationally, many families in turn see congenital lower lip pits as a family trait and therefore fail to seek specialized care and appropriate treatment. Thus, it is also expected that this study will contribute in the future to the genetic counseling for these families and also will improve the knowledge and qualifications of health professionals who care for them.

\section{Acknowledgment}

The authors would like to thank the National Institute of Population Medical Genetics, Hospital Ophir Loyola and the Graduate Program in Oncology and Medical Science (PPOCM) of the Federal University of Pará (UFPA). This study was supported by the National Institute of Population Medical Genetics (465549/2014-4/Conselho Nacional de Desenvolvimento Científico e Tecnológico - CNPq $88887,136366 / 2017-$ 00/Coodernação de Aperfeiçoamento de Pessoal de Nível Superior - CAPES 17/25510000521-0/Fundação de Amparo à Pesquisa do Estado do Rio Grande do Sul - FAPERGS) and ProRectory of Research and Graduate Program - UFPA (PROPESP). This study was financed in part by the Coordenação de Aperfeiçoamento de Pessoal de Nível Superior Brasil (CAPES) - Finance Code 001.

\section{Conflicts of interest}

The authors declare no competing interest.

\section{References}

[1] Cleft lip - a comprehensive review. Shkoukani MA, Chen M, Vong A. 
Front Pediatr. 2013 Dec. 53 (1): 1 - 10. PMID: 2440029.

[2] Van der Woude syndrome: clinical presentation in 64 patients. Huang JJ, Hou JW, Tan YC, Chen KT, Lo L, Chen YR. Cleft Palate Craniofac J. 2007 Nov;44(6):649-52. PMID: 18177185.

[3] Van der Woude syndrome: a review of 11 cases seen at the Lagos University Teaching Hospital. James O, Adeyemo WL, Emeka Cl, Ogunlewe MO, Ladeinde AL, Butali A. Afr J Paediatr Surg. 2014 Jan-Mar;11(1):525. PMID: 24647295.

[4] Van der Woude syndrome: a review. Cardinal signs, epidemiology, associated features, differential diagnosis, expressivity, genetic counselling and treatment. Rizos $\mathrm{M}$, Spyropoulos MN. Eur J Orthod. 2004 Feb;26(1):17-24. PMID: 14994878.

[5] The penetrance and variable expression of the Van der Woude syndrome: implications for genetic counseling. Shprintzen RJ, Goldberg RB, Sidoti EJ. Cleft Palate J. 1980 Jan;17(1):52-7. PMID: 6928118.

[6] Epidemiology of Van der Woude syndrome from mutational analyses in affected patients from Pakistan. Malik S, Kakar N, Hasnain S, Ahmad J, Wilcox ER, Naz S. Clin Genet. 2010 Sep;78(3):247-56. PMID: 20184620.

[7] Van der Woude syndrome: dentofacial features and implications for clinical practice. Lam AK, David DJ, Townsend GC, Anderson PJ. Aust Dent J. 2010 Mar;55(1):51-8. PMID: 20415912.
[8] Mutations in IRF6 cause Van der Woude and popliteal pterygium syndromes. Kondo S, Schutte BC, Richardson RJ, Bjork BC, Knight AS, Watanabe Y, Howard E, de Lima RL, Daack-Hirsch S, Sander A, McDonaldMcGinn DM, Zackai EH, Lammer EJ, Aylsworth AS, Ardinger HH, Lidral AC, Pober BR, Moreno L, Arcos-Burgos M, Valencia C, Houdayer C, Bahuau M, Moretti-Ferreira D, Richieri-Costa A, Dixon MJ, Murray JC. Nat Genet. 2002 Oct;32(2):285-9. PMID: 12219090.

[9] An etiologic regulatory mutation in IRF6 with loss- and gain-of-function effects. Fakhouri WD, Rahimov F, Attanasio C, Kouwenhoven EN, Ferreira De Lima RL, Felix TM, Nitschke L, Huver D, Barrons J, Kousa YA, Leslie E, Pennacchio LA, Van Bokhoven $\mathrm{H}$, Visel A, Zhou H, Murray JC, Schutte BC. Hum Mol Genet. 2014 May 15;23(10):2711-20. PMID: 24442519.

[10] Dominant mutations in GRHL3 cause Van der Woude Syndrome and disrupt oral periderm development. Peyrard-Janvid M, Leslie EJ, Kousa YA, Smith TL, Dunnwald M, Magnusson $M$, Lentz BA, Unneberg P, Fransson I, Koillinen HK, Rautio J, Pegelow M, Karsten A, Basel-Vanagaite L, Gordon W, Andersen B, Svensson T, Murray JC, Cornell RA, Kere J, Schutte BC. Am J Hum Genet. 2014 Jan 2;94(1):23-32. PMID: 24360809.

[11] A preliminary gene map for the Van der Woude syndrome critical region derived from $900 \mathrm{~kb}$ of genomic sequence at 1q32-q41. Schutte BC, Bjork BC, Coppage KB,
Malik MI, Gregory SG, Scott DJ, Brentzell LM, Watanabe Y, Dixon MJ, Murray JC. Genome Res. 2000 Jan;10(1):81-94. PMID: 10645953.

[12] The IRF family of transcription factors: Inception, impact and implications in oncogenesis. Yanai $\mathrm{H}$ Negishi $\mathrm{H}$, Taniguchi $\mathrm{T}$.

Oncoimmunology. 2012 Nov 1;1(8):1376-1386. PMID: 23243601.

[13] Maternal Interferon Regulatory Factor 6 is required for the differentiation of primary superficial epithelia in Danio and Xenopus embryos. Sabel JL, d'Alençon C, O'Brien EK, Van Otterloo E, Lutz K, Cuykendall TN, Schutte BC, Houston DW, Cornell RA. Dev Biol. 2009 Jan 1;325(1):249-62. PMID: 19013452.

[14] Abnormal skin, limb and craniofacial morphogenesis in mice deficient for interferon regulatory factor 6 (Irf6). Ingraham CR, Kinoshita A, Kondo S, Yang B, Sajan S, Trout KJ, Malik MI, Dunnwald M, Goudy SL, Lovett M, Murray JC, Schutte BC. Nat Genet. 2006 Nov;38(11):1335-40. PMID: 17041601.

[15] IRF family of transcription factors as regulators of host defense.

Taniguchi T, Ogasawara K, Takaoka A, Tanaka N. Annu Rev Immunol. 2001;19: 623-55. PMID: 11244049.

[16] Interferon Regulatory Factor 6 Is Necessary for Salivary Glands and Pancreas Development. Metwalli KA, Do MA, Nguyen K, Mallick S, Kin K, Farokhnia N, Jun G, Fakhouri WD. J Dent Res. 2018 Feb;97(2):226-236. PMID: 28898113. 
[17] Genealogical data in population medical genetics: Field guidelines.Poletta FA, Orioli IM, Castilla EE. Genet Mol Biol. 2014 Mar;37(1 Suppl):171-85. PMID: 24764752.

[18] Protein structure analysis of mutations causing inheritable diseases. An e-Science approach with life scientist friendly interfaces.

Venselaar H, Te Beek TA, Kuipers RK, Hekkelman ML, Vriend G. BMC Bioinformatics. 2010 Nov 8;11:548. PMID: 21059217.

[19] PROVEAN web server: a tool to predict the functional effect of amino acid substitutions and indels. Choi $Y$, Chan AP. Bioinformatics. 2015 Aug 15;31(16):2745-7. PMID: 25851949.

[20] SIFT web server: predicting effects of amino acid substitutions on proteins. Sim NL, Kumar P, Hu J, Henikoff S, Schneider G, Ng PC. Nucleic Acids Res. 2012 Jul;40(Web Server issue):W452-7. PMID: 22689647.

[21] Predicting functional effect of human missense mutations using PolyPhen-2. Adzhubei I, Jordan DM, Sunyaev SR. Curr Protoc Hum Genet. 2013 Jan; Chapter 7:Unit7.20. PMID: 23315928.

[22] Novel IRF6 mutations in families with Van Der Woude syndrome and popliteal pterygium syndrome from sub-Saharan Africa. Butali A, Mossey PA, Adeyemo WL, Eshete MA, Gaines LA, Even D, Braimah RO, Aregbesola $\mathrm{BS}$, Rigdon JV, Emeka Cl, James O, Ogunlewe MO, Ladeinde AL, Abate F,
Hailu T, Mohammed I, Gravem PE, Deribew M, Gesses M, Adeyemo AA, Murray JC. Mol Genet Genomic Med. 2014 May;2(3):254-60. PMID: 24936515.

[23] Clinical and genetic features of Van der Woude syndrome in two large families in Brazil. Martelli-Junior $\mathrm{H}$, Chaves MR, Swerts MS, de Miranda RT, Bonan PR, Coletta RD. Cleft Palate Craniofac J. 2007 May;44(3):239-43. PMID: 17477759.

[24] Congenital Symmetrical Lower Lip Pits: Van der Woude Syndrome. Ibrahim A, Ajike S. Oman Med J. 2015 Jan;30(1):e081. PMID: 30838101.

[25] Wound complications after cleft repair in children with Van der Woude syndrome. Jones JL, Canady JW, Brookes JT, Wehby GL, L'Heureux J, Schutte BC, Murray JC, Dunnwald M. J Craniofac Surg. 2010 Sep;21(5):1350-3. PMID: 20856020.

[26] The genetics of isolated orofacial clefts: from genotypes to subphenotypes. Jugessur A, Farlie PG, Kilpatrick N. Oral Dis. 2009 Oct;15(7):437-53. PMID: 19583827.

[27] Gene/environment causes of cleft lip and/or palate. Murray JC. Clin Genet. 2002 Apr;61(4):248-56. PMID: 12030886 .

[28] Identification of IRF6 gene variants in three families with Van der Woude syndrome. Tan EC, Lim EC, Yap SH, Lee ST, Cheng J, Por YC, Yeow
V. Int J Mol Med. 2008 Jun;21(6):74751. PMID: 18506368.

[29] Prevalence and nonrandom distribution of exonic mutations in interferon regulatory factor 6 in 307 families with Van der Woude syndrome and 37 families with popliteal pterygium syndrome. de Lima RL, Hoper SA, Ghassibe M, Cooper ME, Rorick NK, Kondo S, Katz L, Marazita ML, Compton J, Bale S, Hehr U, Dixon MJ, Daack-Hirsch S, Boute O, Bayet B, Revencu N, Verellen-Dumoulin $C$, Vikkula $M$, Richieri-Costa A, Moretti-Ferreira D, Murray JC, Schutte BC. Genet Med. 2009 Apr;11(4):241-7. PMID: 19282774.

[30] Familial non-syndromic cleft lip and palate--analysis of the IRF6 gene and clinical phenotypes. Pegelow $M$, Peyrard-Janvid M, Zucchelli M, Fransson I, Larson O, Kere J, Larsson C, Karsten A. Eur J Orthod. 2008 Apr;30(2):169-75. PMID: 18209213.

[31] Novel mutations in the IRF6 gene for Van der Woude syndrome. Wang X, Liu J, Zhang H, Xiao M, Li J, Yang C, Lin X, Wu Z, Hu L, Kong X. Hum Genet. 2003 Oct;113(5):382-6. PMID: 12920575.

[32] Van der woude syndrome with short review of the literature.

Deshmukh PK, Deshmukh K, Mangalgi A, Patil S, Hugar D, Kodangal SF. Case Rep Dent. 2014; 2014:871460. PMID: 25050184.

[33] Hypoplasia and hypodontia in Van der Woude syndrome. Oberoi S, Vargervik K. Cleft Palate Craniofac J. 
Vol 9 No 1 (2021) DOI 10.5195/d3000.2021.164

2005 Sep;42(5):459-66. PMID:

16149825.

[34] Dental agenesis: genetic and clinical perspectives. De Coster PJ, Marks LA, Martens LC, Huysseune A. J Oral Pathol Med. 2009 Jan;38(1):1-17. PMID: 18771513. 